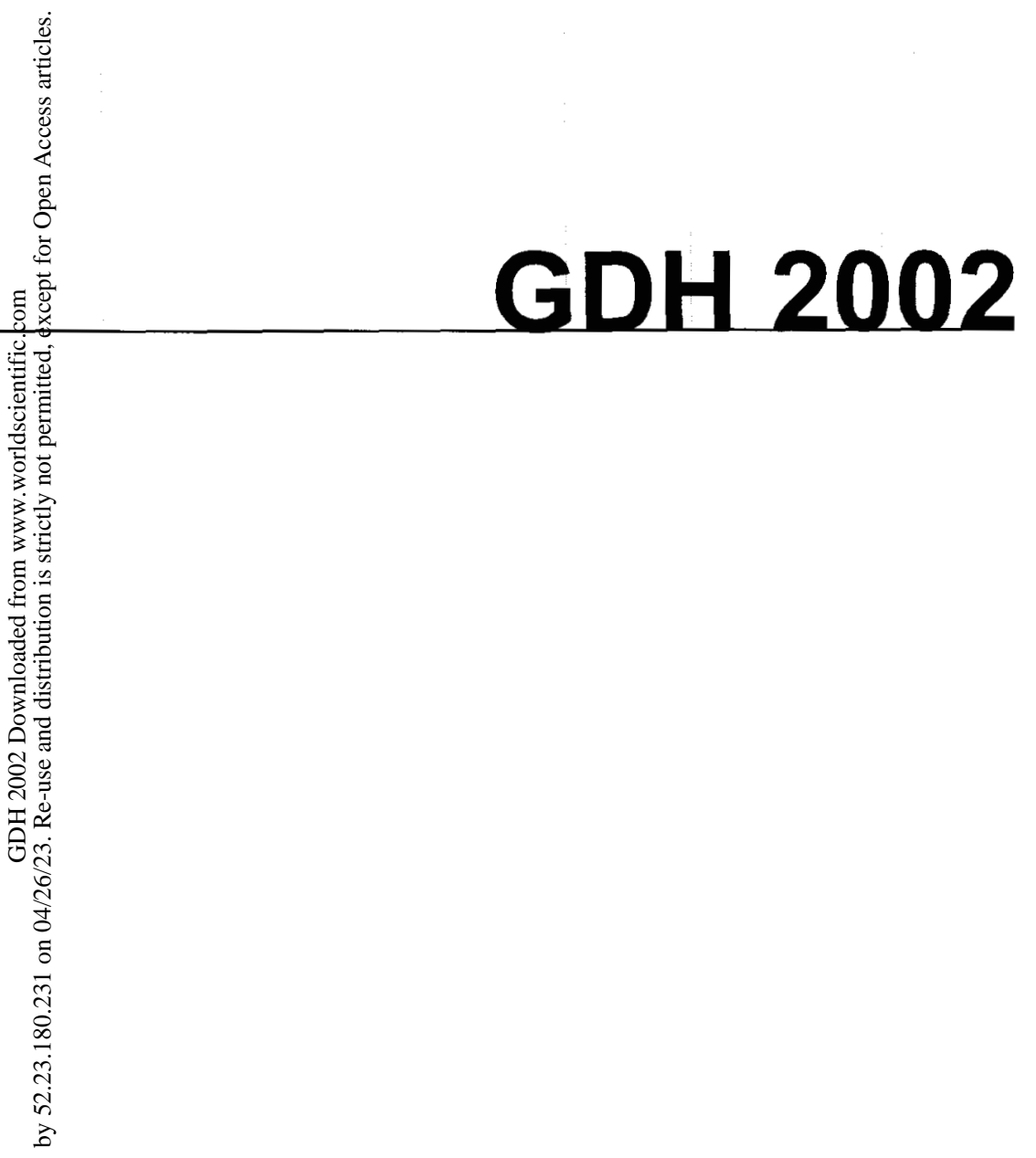


This page intentionally left blank 
Genova, Italy $3-6$ July 2002

\section{Proceedings of the Second International Symposium}

on the Gerasimov-Drell-Hearn Sum Rule and the Spin Structure of the Nucleon

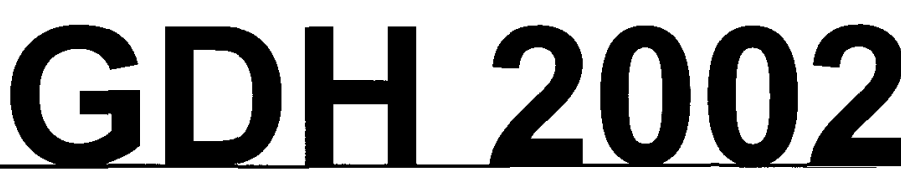

Editors

M. Anghinolfi

M. Battaglieri

R. De Vita

Istituto Nazionale di Fisica Nucleare, Genova, Italy 


\section{Published by}

World Scientific Publishing Co. Pte. Ltd.

5 Toh Tuck Link, Singapore 596224

USA office: Suite 202, 1060 Main Street, River Edge, NJ 07661

UK office: 57 Shelton Street, Covent Garden, London WC2H 9HE

\section{British Library Cataloguing-in-Publication Data}

A catalogue record for this book is available from the British Library.

\section{GDH 2002}

Proceedings of the Second International Symposium on the Gerasimov-Drell-Hearn Sum Rule and the Spin Structure of the Nucleon

Copyright $\odot 2003$ by World Scientific Publishing Co. Pte. Ltd.

All rights reserved. This book, or parts thereof, may not be reproduced in any form or by any means, electronic or mechanical, including photocopying, recording or any information storage and retrieval system now known or to be invented, without written permission from the Publisher.

For photocopying of material in this volume, please pay a copying fee through the Copyright Clearance Center, Inc., 222 Rosewood Drive, Danvers, MA 01923, USA. In this case permission to photocopy is not required from the publisher.

ISBN $981-238-397-2$ 


\title{
GDH 2002
}

\section{2nd International Symposium on the Gerasimov-Drell-Hearn Sum Rule and the Spin Structure of the Nucleon}

\author{
Genova, Italy \\ July 3-6, 2002
}

\section{Organization}

\author{
Conference Chairmen \\ M. Anghinolfi (Genova), J. P. Chen (Jefferson Lab), \\ and D. Drechsel (Mainz)
}

\section{Organizing Committee}

H. J. Arends (Mainz), M. Anghinolfi (Genova), M. Battaglieri (Genova), J. P. Chen (Jefferson Lab), R. De Vita (Genova), D. Drechsel (Mainz), S. Kuhn (ODU), P. Pedroni (Pavia), M. Taiuti (Genova)

\section{Advisory Commitee}

M. Anselmino (Torino), N. Bianchi (Frascati), P. Bosted (SLAC), V. Burkert (Jefferson Lab), A. D'Angelo (Roma), K. de Jager (Jefferson Lab), M. Giannini (Genova), B. Hersmann (UNH), N. Horikawa (Nagoya), Z. Meziani (Temple), T. Nakano (Osaka), O. Rondon-Aramayo (UVA), A. Sandorfi (BNL), B. Schoch (Bonn), R. Van de Vyver (Gent), T. Walcher (Mainz), W. Weise (Munchen)

\section{Institutional Sponsors}

Istituto Nazionale di Fisica Nucleare

Jefferson Laboratory

Università di Genova 
This page intentionally left blank 


\section{Foreword}

This volume contains the proceedings of the 2nd International Symposium on the Gerasimov-Drell-Hearn sum rule and the Spin Structure of the Nucleon, which was held in Genova during July 3-6, 2002. This conference followed the first venue of June 2000 in Mainz and had the purpose of bringing together experimental and theoretical physicists working in this field.

In the last three decades, the study of the spin structure of the nucleon has been one of the main topics in hadronic physics. A large wealth of information was obtained by Deep Inelastic Scattering experiments performed at SLAC and CERN. However, only recently it was realized that a full understanding of the nucleon spin requires a detailed study of the helicity structure from the high momentum transfer region. i.e. the perturbative domain, down to the low momentum transfer region, the realm of non perturbative QCD. In the last years this regime has been investigated at several facilities all over the world both with photon and electron beams, leading to a renewed interest of theoretical and experimental physicists in these topics.

It was the aim of this Symposium to offer the possibility of presenting and discussing the latest results in this field and to indicate the main issues to be addressed in the future. Indeed the enthusiastic response of the community, with a strong international participation to the Symposium, was an indication that this field of physics is lively and rapidly developing.

It is our pleasure to acknowledge the support of the institutional sponsors that made this conference possible: the Italian Istituto Nazionale di Fisica Nucleare, the Thomas Jefferson National Accelerator Facility, and the Università di Genova. We also gratefully acknowledge the advice from the members of the International Advisory Committee. Our special thanks go to all the speakers and conveners who contributed to the success of the meeting. The Symposium would not have been possible without the organizational efforts of many people, the secretaries, the students, and the technicians, to all of which we express our gratitude. We also want to thank the Maestro Flavio Constantini, who kindly permitted the use of his original work of art as a poster for the Symposium.

Genova, February 2002

M. Anghinolfi, M. Battaglieri, R. De Vita 


\section{CONTENTS}

\section{Introduction}

Introduction: GDH and Related Topics

M. M. Giannini

Quark-Hadron Duality in Photoabsorption Sum Rules and Two Photon Decays of Meson Resonances

S. B. Gerasimov

What Do We Know about the Proton Spin Structure?

M. Anselmino

\section{Theory}

MAID and the GDH Sum Rule in the Resonance Region

L. Tiator

Interplay of Hard and Soft Processes at JLab Energies

A. Afanasev and C. E. Carlson

Generalized Parton Distributions and Sum Rules

M. Vanderhaeghen

Proton and Neutron Polarized Structure Functions

from Low to High $Q^{2}$

S. Simula, M. Osipenko, G. Ricco, and M. Taiuti

Chiral Dynamics and the Spin Structure of the

Nucleon at Low Energies

T. R. Hemmert

Structure Functions on the Lattice

M. Guagnelli, F. Palombi, R. Petronzio, K. Jansen,

A. Shindler, and I. Wetzorke

Transversity: An Update

V. Barone 


\section{Experiments}

Experimental Results for the GDH Sum Rule on the

Proton from Pion Threshold to $3 \mathrm{GeV}$

P. Grabmayr for the GDH-Collaboration

Helicity Dependence of Exclusive Pion Photo-Production in the First and Second Resonance Region

A. Braghieri for GDH and A2 Collaborations

Neutron Spin-Structure Studies at Jefferson Lab Hall A

G. D. Cates

Double Spin Asymmetries in Electron-Nucleon

Scattering in Halls B and $C$ at JLAB

D. G. Crabb

Study of Nucleon Resonances with Polarization

Degrees of Freedom at MIT/Bates

T. Botto for the OOPS and BLAST Collaborations

Spin Physics at HERMES

A. Fantoni for the HERMES Collaboration

GDH at GRAAL with Polarized $H D$ Target

J.-P. Didelez for the GRAAL Collaboration

First Photo-Pion Double Polarization Experiments

Using Polarized HD at LEGS

A. M. Sandorfi for the LEGS Collaboration

Experimental Tests of the GDH and Other Sum

Rules at SLAC

P. E. Bosted

Status of SPring-8 LEPS Project and the GDH Experiment

T. Iwata
A Study of the GDH Sum Rule Integrand for the Deuteron at TUNL and $H I \gamma S$ Near Photodisintegration Threshold

H. R. Weller for the TUNL and the GDH Collaborations 


\section{Instrumentation and Future Projects}

\section{Progress in Scintillating Polarized Targets for}

Spin Physics

183

B. van den Brandt, P. Hautle, J. A. Konter,

and E. I. Bunyatova

Polarized Targets for the CLAS Detector at Jefferson Lab

189

C. D. Keith on behalf of the CLAS Collaboration

The JLAB Polarized ${ }^{3} \mathrm{He}$ Target

195

K. Slifer for the Jefferson Lab Hall A Collaboration

High Accuracy Polarization and Density Measurement of a Solid State Polarized Target

CH. Rohlof, and H. Dutz

The Hermes Recoil Detector

I. M. Gregor on behalf of the Hermes Collaboration

Future Plans for the Mainz Real Photon Facility

213

A. Thomas for the A2-Collaboration

Two Septum Magnets for Forward Angle Physics at Jlab

217

G. M. Urciuoli, F. Cusanno, S. Frullani, F. Garibaldi, M. Iodice,

G. De Cataldo, R. De Leo, L. Lagamba, P. Brindza, J. P. Chen,

S. Lassiter, J. J. Lerose, and A. Deur

The Crystal Ball for Future Experiments at MAMI

A. Starostin

\section{Spin Structure Functions in Nucleons and Nuclei}

Neutron Spin Structure Function Results from

Jefferson Lab Hall A

229

N. Liyanage

The $Q^{2}$ Dependence of the Deuteron Spin Structure

Function $g_{1}^{d}$ at CEBAF

M. Taiuti for the CLAS Collaboration 
Spin Structure of the Neutron at Low $Q^{2}$

K. Slifer for the Jefferson Lab Hall A Collaboration

Precision Measurement of the Nucleon Spin Structure Functions in the Region of the Nucleon Resonances

J. Yun for the Resonance Spin Structure (RSS) Collaboration

Measurement of the Helicity Asymmetry of the $\gamma p$

Cross Section Between 2.5 and 5.3 GeV

D. I. Sober, L. Todor, P. E. Bosted, S. E. Kuhn, and the CLAS

Collaboration at Jefferson Lab

Recent Measurements of Longitudinal and Transverse

Unpolarized Structure Functions, and Their Impact on Spin Asymmetry Measurements

M. E. Christy

The Proton Structure Function $F_{2}$ in the Resonance Region

M. Osipenko, G. Ricco, M. Taiuti, M. Anghinolfi, M. Battaglieri,

R. De Vita, M. Ripani, S. Simula, G. Fedotov, E. Golovach,

B. Ishkhanov, V. Mokeev, and the CLAS Collaboration

\section{Helicity Amplitudes, Resonance Structure, and Spin Polarizabilities}

Resonance Structure and Polarizability of the Nucleon

D. Drechsel

Helicity Amplitudes in the Hypercentral Quark Model

M. M. Giannini, E. Santopinto, and A. Vassallo

SAID Results for GDH-Related Sum Rules

R. A. Arndt, W. J. Briscoe, I. I. Strakovsky, and R. L. Workman

The Commissioning of the Hall-B Beamline of Jefferson Lab for Coherently Producing a Beam of Linearly-Polarized Photons

P. L. Cole, J. Kellie, F. J. Klein, K. Livingston, J. A. Mueller,

J. C. Sanabria, and D. J. Tedeschi, cospokespersons of

g8 for the CLAS Collaboration 
Study of the $\Delta(\mathbf{1 2 3 2})$ using Double-Polarization Asymmetries

A. Biselli and J. Kuhn for the CLAS Collaboration

Electron Beam Asymmetry Measurements from

Exclusive $\pi^{0}$ Electroproduction in the $\Delta(\mathbf{1 2 3 2})$

Resonance Region

303

K. Joo for the CLAS Collaboration

Results of the Helicity-Dependent Double-Pion

Photoproduction on the Proton at MAMI

M. Lang for the A2- and GDH-Collaborations

Theoretical Study of Polarization Observables in Double Pion Photoproduction on the Proton

L. Roca, J. C. Nacher, E. Oset, and M. J. Vicente Vacas

Polarization Degrees of Freedom in Charged Double

Pion Production by Virtual Photons

321

E. Golovach, V. Burkert, V. Mokeev, M. Ripani, M. Anghinolf,

M. Battaglieri, A. Boluchevskii, G. Fedotov, R. De Vita,

B. Ishkhanov, N. Markov, M. Osipenko, G. Ricco, N. Shvedunov, and M. Taiuti

Omega Photoproduction at GRAAL

A. d'Angelo for the GRAAL Collaboration

Fixed- $t$ and Fixed-Angle Dispersion Relations for

Real Compton Scattering

B. Pasquini, D. Drechsel, and M. Vanderhaeghen

Compton Scattering at GRAAL

A. Fantini for the GRAAL Collaboration

Spin Polarizabilities and Polarizabilities of the Nucleon Studied by Free and Quasi-Free Compton

Scattering at MAMI (Mainz)

M. Schumacher

The Generalized Spin Polarizabilities of Nucleon in HBChPT at $\mathcal{O}\left(p^{4}\right)$

C. W. Kao and M. Vanderhaeghen 


\section{Spin and Hard Scattering}

Spin and Hard Processes

P. Kroll

Proton Polarization in Neutral Pion Photo-Production

K. Wijesooriya for the Jefferson Lab Hall A Collaboration

Polarization Measurements in Deuteron

Photodisintegration

373

K. McCormick for the Jefferson Lab Hall A Collaboration

Polarization Observables and Forward-Backward

Asymmetry in High-Energy Deuteron

Photodisintegration within the Quark-Gluon Strings

Model

V. Yu. Grishina, L. A. Kondratyuk, W. Cassing, E. De Sanctis, M. Mirazita, F. Ronchetti, and P. Rossi

Real Photon Scattering on the Proton

C. W. de Jager and B. Wojtsekhowski for the JLAB

Hall A Collaboration

Transversity at HERMES

391

V. Muccifora for the HERMES Collaboration

Accessing Transversity with Interference

Fragmentation Functions

397

M. Radici

Polarization Phenomena by Deuteron Fragmentation into Pions

A. Yu. Illarionov, A. G. Litvinenko, and G. I. Lykasov

\section{Conclusion}

Outlook

B. H. Schoch 


\section{Appendix}

$\begin{array}{ll}\text { Author Index } & 413\end{array}$

$\begin{array}{ll}\text { Program of the Symposium } & 415\end{array}$

$\begin{array}{ll}\text { List of Participants } & 421\end{array}$ 